\title{
A public gene trap resource for mouse functional genomics
}

\section{To the editor:}

Gene trapping is a high-throughput approach that can be used to introduce insertional mutations across the genome in mouse embryonic stem (ES) cells. Gene trap vectors simultaneously mutate and report the expression of the endogenous gene at the site of insertion and provide a DNA tag for the rapid identification of the disrupted gene. The generation of mutant mice from a large collection of ES cell lines carrying gene trap insertions could be applied to large-scale functional analysis of the $\sim 30,000$ mammalian genes. The overall impact of gene trap resources will rest on the fraction of the genome that is accessible with this technology, the efficiency relative to other competing technologies and the availability of such a resource to the academic community. Lexicon Genetics, a US-based biotechnology company, was the first to implement a genome-wide gene trapping program ${ }^{1}$ and has developed OmniBank (http://www.lexicon-

genetics.com), the largest library of mutant ES cell lines. A parallel effort was initiated in the public domain by several academic groups in the International Gene Trap Consortium (IGTC; http://www.igtc.ca). The recent release of OmniBank sequence tags to GenBank ${ }^{2}$ has made it possible to compare the size and efficiency of the existing gene trap libraries.

We confirm that Lexicon achieved close to $60 \%$ coverage of the genome from 200,000 OmniBank sequence tags deposited in GenBank (Fig. 1). Our analysis, supported independently by Lexicon $^{3}$, indicates that the rate of trapping new genes was not linear but declined within the first 100,000 tags to a rate at which 1 new gene was added every 35 tags, comparable to the efficiency of high-throughput gene targeting methods ${ }^{4}$. To date, the IGTC has attained 32\% genome coverage in 27,000 tags; trapping

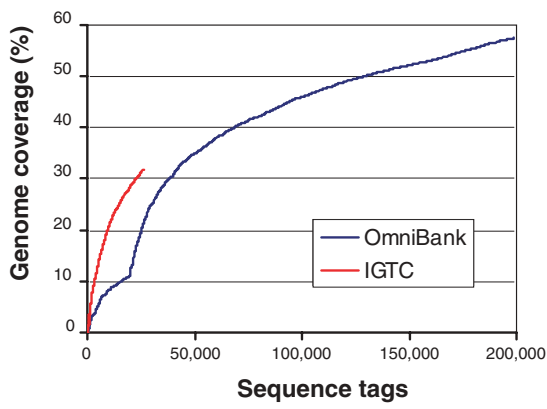

Figure 1 Comparison of the rates of trapping of the IGTC and OmniBank resources. Unique Ensembl genes were identified using MAPTAG (http://www.sanger.ac.uk/Software/MAPTAG), an automated annotation program that identifies short, almost perfect matches to gene features in Ensembl. An additional 10\% of the trapped genes were identified from BLAST searches $\left(\leq \mathrm{E}^{-04}\right)$ of the RefSeq database. Genome coverage was calculated as the fraction of 8,000 full-length 'sentinel' genes ${ }^{2}$ trapped in each resource.

is likewise nonlinear, but the initial rate seems to be somewhat faster than Lexicon's (Fig. 1). The seemingly higher efficiency may relate to the diversity of plasmid and retroviral vector designs used by the IGTC that could help overcome insertion site preferences of any single vector ${ }^{5}$; further studies are needed to fully understand how vector design and other experimental factors influence the efficiency of gene trapping. One-fifth of the genes trapped by the IGTC were not represented in the sequence tags released by Lexicon (Supplementary Tables 1-3 online). Thus, the two efforts together have trapped nearly two-thirds of all genes in mice. We conclude that gene trapping is an effective strategy to mutate a substantial fraction of the genes in mice that compares favorably with gene-targeting approaches.

Furthermore, we continue to refine the technology, particularly in developing strategies for postinsertional modification of the trapped loci to create a wide range of desired alleles. The IGTC will provide an important public resource of new mutations in mice that will accelerate the pace of functional annotation of the mammalian genome.

Gene trap cell lines generated by the IGTC are available without restriction (http://baygenomics.ucsf.edu; http://www. genetrap.de; http://www.escells.ca; http://www.sanger.ac.uk/genetrap; http://www.fhcrc.org/labs/soriano/GTdb; http://www.cmhd.ca) and all sequence tags are mapped on the Ensembl mouse genome browser http://www.ensembl.org/ Mus_musculus/; select DAS Source 'GeneTrap').

Note: Supplementary information is available on the Nature Genetics website.

International Gene Trap Consortium William C Skarnes ${ }^{1}$, Harald von Melchner ${ }^{2}$, Wolfgang Wurst ${ }^{3}$, Geoff Hicks ${ }^{4}$, Alex S Nord ${ }^{1}$, Tony Cox ${ }^{1}$, Stephen G Young ${ }^{5}$, Patricia Ruiz ${ }^{6}$, Phil Soriano ${ }^{7}$, Marc Tessier-Lavigne ${ }^{8}$, Bruce R Conklin ${ }^{5}$, William L Stanford ${ }^{9}$ \&Janet Rossant ${ }^{10}$

${ }^{1}$ Wellcome Trust Sanger Institute, Wellcome Trust Genome Campus, Hinxton, Cambridge, CB10 1SA, UK. ${ }^{2}$ Laboratory for Molecular Hematology, University of Frankfurt Medical School, Frankfurt am Main, Germany. ${ }^{3}$ Institute of Developmental Genetics, GSF Research Center, Neuherberg, Germany, and Max Planck Institute of Psychiatry, Munich, Germany. ${ }^{4}$ Manitoba Institute of Cell Biology, Department of Biochemistry \& Medical Genetics, Faculty of Medicine, University of Manitoba, Winnipeg, Canada. ${ }^{5}$ Gladstone Institute of Cardiovascular Disease, Department of Medicine, University of California San Francisco, California, USA. ${ }^{6}$ Max-Planck Institute for Molecular Genetics, Center for Cardiovascular Research, Berlin, Germany. ${ }^{7}$ Program in Developmental Biology, Division of Basic Sciences, Fred Hutchinson Cancer Research Center, Seattle, Washington, USA. ${ }^{8}$ Genetech, South San Francisco, 
California, USA. ${ }^{9}$ Institute of Biomaterials \& Bioengineering and ${ }^{10}$ Samuel Lunenfeld Research Institute, Department of Medical Genetics and Microbiology, University of

Toronto, Toronto, Canada. Correspondence should be addressed to W.C.S.

(skarnes@sanger.ac.uk).

1. Zambrowicz, B.P. et al. Nature 392, 608-611 (1998).

2. Zambrowicz, B.P. et al. Proc. Natl. Acad. Sci. USA 100, 14109-14114 (2003).
3. Zambrowicz, B.P. et al. Proc. Natl. Acad. Sci. USA 101, 4332-4333 (2004).

4. Valenzuela, D.M. et al. Nat. Biotechnol. 21, 652-659 (2003).

5. Hansen, J. et al. Proc. Natl. Acad. Sci. USA 100, 9918-9922 (2003)

\section{Inauguration of the African Society of Human Genetics}

\section{To the editor:}

In 2003, the African Society of Human Genetics (AfSHG) was established. The aim of AfSHG is to equip the African scientific community and policy-makers with the information and practical knowledge they need to contribute to the field of genetics research and to attract global attention to the efforts of African scientists. An important goal of this organization is to provide opportunities for networking and collaboration among professionals working on genetic and genomic issues relevant to Africa. By achieving these goals, AfSHG will help diminish the widening gap between Africa and the Western world in biomedical science.

The inaugural conference of AfSHG was held 8-9 December 2003 in Accra, Ghana. The theme for the meeting was

"Biomedical Research in Africa with Emphasis on Genetics". The meeting began with a review of socioeconomic and health conditions on the African continent. The importance of current realities for building a strong foundation for developing genetics and genomics in Africa was considered.

Genomic variation in the African diaspora was explored with attention to understanding the differential distribution of diseases, including malaria, tuberculosis, AIDS, diabetes, hypertension and cancer. Challenges surrounding the design and implementation of the only biobank in Africa, the Gambia National DNA Collections, were described. Current and potential applications of bioinformatics for the expansion of genetics and genomic research in Africa were reviewed. Geneenvironment interaction and its impact on the distribution of common complex diseases in Africa and the African diaspora were critically assessed. Ethical issues surrounding voluntary participation and informed consent in genetic research conducted in African settings and the current status of bioethics in medical education were discussed.
Future challenges for AfSHG. The fundamental goals of AfSHG are building capacity and networking. AfSHG plans to implement these objectives through several specific aims: (i) Information dissemination. The annual AfSHG conference and additional specialized workshops will provide a forum for disseminating and exchanging information through lectures and interactive training programs. (ii) Mentoring networks and educational resources. AfSHG will cultivate interest in investigative science and encourage African undergraduate and graduate students to pursue careers related to human genetics and biomedical sciences in general. (iii) Genetic advocacy. AfSHG will give voice to the cause of human genetics in Africa. AfSHG members will interface with policy-makers, the media and the public to help reduce fears and concerns surrounding genetics research. AfSHG will promote the design and implementation of genetics research that is ethically and culturally acceptable. (iv) Development of online courses. Members of AfSHG will develop online courses through affiliations with African, American and European universities and assist members with access to the internet. Additionally, programs will be initiated to diminish the widening North-South divide in biomedical science and technology. AfSHG will facilitate the participation of African scientists at annual international meetings of other professional organizations for genetics. (v) African Journal of Human Genetics. A scientific journal including research reports and critical reviews will be established to address specific interests of professionals working on genetics in the African context. This journal will supplement information available in textbooks and other resources, which are often scarce at many African institutions. (vi) Collaborative research. AfSHG will encourage and enhance collaborative research, which is currently responsible for $50 \%$ of the productivity of the African scientific community ${ }^{1}$. AfSHG will bring together foreign scientists working in Africa and African investigators to present information about populations of individuals with specific diseases, databases and technologies that would generate collaborative research. In addition to providing greater opportunities for research and exploration of genetics, applications for research grants and information about research sponsors will be made available. Courses in successful grant writing and training in clinical research methods, data management and manuscript development will be developed.

Membership in AfSHG is open to any individual who is interested in issues of human genetics in Africa, including but not limited to issues of education, clinical care, research, public health, ethics, law and policy. The field of genetics represents a continually expanding global enterprise. Successful international application of scientific and technological knowledge associated with genetics depends on cooperative arrangements between professionals living in diverse African countries and between Africans and their counterparts residing in other areas of the world. Collaborative partnerships in genomics and genetics will contribute greatly to promoting a sustained commitment to the development of the field of genetics in Africa. We envision AfSHG as the organization that will be a driving force in building a strong foundation for scientists and scholars working in genomics and genetics issues in Africa. Information on the AfSHG is available at http://www.afshg.org/.

\section{Charles N Rotimi}

National Human Genome Center, College of Medicine, Howard University, 2216 6th Street, NW, Suite 206, Washington, DC 20059, USA.

1. Jaffe, S. Back to Africa (Yes, it's possible to do science in Africa, but it's not that easy). The Scientist 16, 48-50 (2003). 